\title{
Impact of depressive disorders on quality of life after middle ear surgery in patients with chronic otitis media
}

\author{
Susen Lailach ${ }^{1}\left[\right.$ Theresa Langanke $^{1} \cdot$ Thomas Zahnert $^{1} \cdot$ Susan Garthus-Niegel ${ }^{2,3,4} \cdot$ Marcus Neudert $^{1}$
}

Received: 14 July 2020 / Accepted: 23 September 2020 / Published online: 3 October 2020

(c) The Author(s) 2020

\begin{abstract}
Purpose The aim of this study was to determine whether preoperative depressive symptoms influence health-related quality of life (HRQOL) after middle ear surgery in patients with chronic otitis media (COM).

Methods This prospective clinical case study was conducted at a tertiary referral center. All 102 patients who had undergone middle ear surgery for COM were assessed clinically and by audiometric testing (pure tone audiometry) in pre- and postoperative settings. Disease-specific HRQOL was assessed by the validated chronic otitis media outcome test 15 (COMOT-15) and the Zurich chronic middle ear inventory (ZCMEI-21). General HRQOL was measured using the short form 36 (SF-36). Depressive symptoms were assessed using the patient health questionnaire (PHQ-D). The Charlson comorbidity index (CCI) was used to classify comorbidities. The middle ear status was determined using the ossiculoplasty outcome parameter staging (OOPS) index.

Results After middle ear surgery, the total COMOT-15 and ZCMEI-21 scores improved significantly $(p<0.001)$. General HRQOL (total SF-36 score) was unaffected by surgery $(p<0.05)$. Patients without elevated depressive symptoms had significantly better total scores for the COMOT-15 $(p<0.01)$, ZCMEI-21 $(p<0.001)$, and for SF-36 $(p<0.001)$ postoperatively. The results of the multiple regression analyses show that, after adjusting for the OOPS, CCI, and hearing improvement, preoperative depressiveness was significantly associated with worse postoperative COMOT-15 and ZCMEI-21 outcome scores $(\beta=0.425$ and $\beta=0.362, p<0.001)$.
\end{abstract}

Conclusion Preoperative depressiveness was an essential predictive factor for HRQOL in patients with COM. This should be considered during patient selection to provide more suitable preoperative counseling.

Keywords Depressiveness $\cdot$ Tympanoplasty $\cdot$ Cholesteatoma $\cdot$ Quality assessment $\cdot$ Middle ear reconstruction

\section{Introduction}

Susen Lailach

Susen.Lailach@uniklinikum-dresden.de

1 Faculty of Medicine Carl Gustav Carus, Department of Otorhinolaryngology, Head and Neck Surgery, Technische Universität Dresden, Fetscherstraße 74, 01307 Dresden Saxony, Germany

2 Department of Medicine, Faculty of Human Sciences, Medical School Hamburg, Hamburg, Germany

3 Faculty of Medicine Carl Gustav Carus, Institute and Policlinic of Occupational and Social Medicine, Technische Universität Dresden, Fetscherstraße 74, 01307 Dresden, Saxony, Germany

4 Department of Child Health and Development, Norwegian Institute of Public Health, Oslo, Norway
Chronic otitis media (COM) is a common disease that affects $0.45-2.6 \%$ of the population $[1,2]$. Patients with COM suffer from ear discharge, pain, and hearing impairment; due to extensive communication problems, this results in social and emotional restrictions. Additionally, COM leads to restrictions in daily activities and social interactions and increased use of the healthcare system [3-6].

For several years now, health-related quality of life (HRQOL) measurements have been an increasingly important outcome parameter even after middle ear surgery [7]. Previous studies mainly focused on the development and validation of measurement instruments as well as the evaluation of pathology-associated or surgery-associated influencing factors [3, 5, 8-11]. Especially in patients who have a reduced disease-specific HRQOL despite good hearing and 
normal ear findings, the extent to which patient-related factors modulate disease-specific HRQOL remains unclear.

Depression has long been associated with hearing disorders [12-14]. However, the coexistence of mood disorders in patients with COM was analyzed in only a few studies [15, 16]. Particularly, studies in orthopedics and pain therapy demonstrated that patients with mental health disorders have been associated with less postoperative improvement [17, 18]. In patient-reported outcome measures (PROMs), patients with anxiety and depression felt that interventions were less beneficial compared to others without these comorbidities [19-22]. Given that depressiveness affects the perception of chronical illness [23-25], depressive disorders may modulate the relationship between COM symptom burden and the decrease in HRQOL in patients with COM.

As healthcare continues its transition to evidence-based medicine, surgeons must identify and analyze individual patient-related factors that affect treatment outcomes. By understanding how patient characteristics affect surgical outcomes, surgeons can achieve more prudent patient selection while providing better preoperative advice.

The aim of this study is to determine whether preoperative depressive symptoms influence PROMs after middle ear surgery for patients with COM.

\section{Patients and methods}

\section{Study design and population}

Between July 2017 and December 2018, 102 patients with COM (with or without cholesteatoma) were prospectively enrolled. They underwent primary or revision surgery at a tertiary referral center. Patients with and without postoperative complications were included. Preoperative assessment was performed 1 day before surgery. Treatment evaluation was performed 6 months postoperatively. Patients who missed the control visit after 6 months were excluded. Preand postoperative evaluation included HRQOL measurement, screening for the presence and severity of depression, registration of comorbidities, and pure tone audiometry.

\section{Surgical technique}

In all patients, a retroauricular incision was used. Tympanic membrane reconstruction was performed via an underlay technique using fascia and cartilage slices. In patients with cholesteatoma, a sequential surgical strategy was preferred, as described previously [11]. All open mastoid cavities were obliterated with autologous bone pate or bone replacement material (bioactive glass S53P4) and sliced concha cartilage plates. The ossicular chain was reconstructed using titanium partial ossicular replacement prostheses (PORP: titanium clip prosthesis, titanium ankle prosthesis, titanium bell prosthesis) or total ossicular replacement prostheses (TORP: Aerial Type Düsseldorf, malleus notch TORP, all Kurz Company, Dusslingen, Germany). Hearing reconstruction and cholesteatoma removal were routinely performed in a single-stage procedure.

\section{HRQOL measurement}

Disease-specific HRQOL measurement was performed using the validated and disease-specific chronic otitis media outcome test 15 (COMOT-15) and the Zurich chronic middle ear inventory (ZCMEI-21) in German language. The short form 36 (SF-36) was used to determine general HRQOL.

\section{COMOT-15}

The COMOT-15 has been previously validated in German language and included 15 Likert scaled items [8]. The survey is subdivided into three subscores: "ear symptoms" (items 1-6), "hearing function" (items 7-9), and "mental health" (items 10-13). From this, an overall score was determined (items 1-13). Additionally, a general evaluation of diseasespecific HRQOL (item 14) and the frequency of physician consultations (item 15) was conducted. As described previously, COMOT-15 scores were normalized to a scale from 0 to 100 by dividing the sum of the raw scores of the items by the sum of spans of the items followed by multiplying by 100 [8]. Higher scores indicated a poorer HRQOL.

II. ZCMEI-21

The ZCMEI-21 has been previously validated in German language [9] and included four subscales: "ear signs and symptoms" (items 1-5), "hearing function" (items 6-10), "psychosocial impact" (items 11-18), and the "use of medical resources" (items 19-21). All answers are presented using a 5-point Likert scale. Higher scores indicated a poorer HRQOL.

III. SF-36

The German version of the SF-36 was validated by Bullinger [26] The SF-36 records eight aspects of subjective health: "physical functioning" (10 items), "role-functioning physical" (4 items), "bodily pain" (2 items), "general health" (5 items), "vitality" (4 items), "social functioning" (2 items), "role-functioning emotional" (3 items), and "mental health" (5 items). Data were scored according to the SF-36 Analysis and Interpretation Manual [27]. The scales were scored from 0 (lowest level of functioning) to 100 (highest level of functioning). 


\section{Depression screening}

The German version of the patient health questionnaire (PHQ-D) [28] was used to assess the severity of depressive symptoms. The PHQ-D has been developed to screen for mental disorders in primary care, using diagnostic criteria from the fourth edition of the American Psychiatric Association 's Diagnostic and Statistical Manual of Mental Disorders (DSM-IV). The depression module (PHQ-9) of the PHQ-D is a screening tool used in numerous patient populations to quantify the severity of patient-reported depressive symptoms. Patients rate the occurrence of depressive symptoms within the last two weeks on a 4-point scale from 0 ("not at all") to 3 ("nearly every day"). The severity of depressive symptoms was analyzed as a continuous score from 0 (none) to 27 (severe). Additionally, the categorical algorithm was applied to distinguish between patients with and without depressive symptoms. For the categorical algorithm, the answers to the questions were dichotomized: "not at all" $(0)$ and "several days" (1) were coded as 0 ("symptom absent"), and the answers "more than half the days" (2) and "nearly every day" (3) were coded as 1 ("symptom present"). According to the PHQ-D manual [28], patients were classified as "depressed" when at least five symptoms were present and at least one symptom was "depressed feelings" or "loss of interest".

\section{Comorbidity and disease-specific parameters}

The Charlson comorbidity index (CCI, Table 1) comprises 17 comorbidities, which are weighted (from 1 to 6) based on the adjusted risk of mortality or resource use [29]. The sum of all the weights results in a single comorbidity score for each patient.

The ossiculoplasty outcome parameter staging (OOPS) index [30] was designed to provide a risk and outcome analysis in the context of the middle ear environment (Table 2). The calculation is based on intraoperative middle ear findings and preoperative examination. The OOPS index was used to evaluate the individual severity of the middle ear disease.

\section{Audiological assessment}

According to the Committee on Hearing and Equilibrium guidelines, pre- and postoperative air conduction (AC) thresholds were calculated as an average (pure tone average, PTA) over the frequencies $0.5,1,2$, and $3 \mathrm{kHz}$ [31].

\section{Statistical analyses}

Descriptive statistics including mean and standard deviation (SD) were used to represent demographic and outcome data. Normality of the outcome scores was assessed
Table 1 Charlson comorbidity index (CCI)

\begin{tabular}{ll}
\hline Comorbid conditions & CCI weights \\
\hline Myocardial infarction & 0 \\
Congestive heart failure & 2 \\
Peripheral vascular disease & 0 \\
Cerebrovascular disease & 0 \\
Dementia & 2 \\
Chronic pulmonary disease & 1 \\
Rheumatic disease & 1 \\
Peptic ulcer disease & 0 \\
Mild liver disease & 2 \\
Diabetes without chronic complications & 0 \\
Diabetes without chronic complications & 1 \\
Paraplegia or hemiplegia & 2 \\
Renal disease & 1 \\
Any malignancy without metastasis & 2 \\
Moderate or severe liver disease & 4 \\
Metastastic malignoma & 6 \\
AIDS /HIV & 4 \\
\hline
\end{tabular}

Table 2 The ossiculoplasty outcome staging (OOPS) index

\begin{tabular}{lll}
\hline Risk factor & & Risk value \\
\hline Drainage & None & 0 \\
& Present $>50 \%$ of the time & 1 \\
Mucosa & Normal & 0 \\
& Fibrotic & 2 \\
Ossicular chain & Normal & 0 \\
& Malleus + & 1 \\
\multirow{4}{*}{ Type of surgery } & Malleus - & 2 \\
& No mastoidectomy & 0 \\
& Intact canal wall mastoideectomy & 1 \\
Revision surgery & Canal wall down mastoidectomy & 2 \\
& No & 0 \\
& Yes & 2 \\
\hline
\end{tabular}

using the Kolmogorov-Smirnov test. For normally distributed outcome scores, a paired $t$ test was used to compare the mean scores pre- and postoperatively. Mean scores between two different groups were analyzed using an independent $t$ test.

Multiple linear regression analyses were conducted to determine the effect of preoperative depressiveness on outcome scores, controlling for comorbidity (CCI), middle ear pathology (OOPS index), and improvement in the AC. A $p$ value $<0.05$ was considered statistically significant. 


\section{Sample size and approval}

The number of patients was based on a statistical sample size calculation (statistical power 0.8 , alpha $=0.05, n=98$ ). Institutional review board approval was obtained before study initiation by the local ethical review committee (EK $166,042,017)$. All patients gave their informed consent. The study was conducted in accordance with the Declaration of Helsinki 1964.

\section{Results}

This study included 102 patients (50 males and 52 females) with a mean age of $49.32 \pm 16.04$ years. Due to the high response rate (102/132 patients, $77 \%)$ and similar gender and age distribution, no response bias was present. Fiftysix $(55 \%)$ patients underwent revision surgery, 62 patients (60.8\%) presented with chronic mesotympanic otitis media, and 40 patients (39.2\%) suffered from cholesteatoma. Eighteen $(17.6 \%)$ patients underwent canal wall down surgery. In $84(82.4 \%)$ patients, an intact canal wall technique was performed.

In total, 44 patients (43.1\%) had an intact ossicular chain. Hearing reconstruction was performed by PORP in 23 (22.5\%) patients and by TORP in 26 patients (25.5\%). Due to middle ear pathology (fixed or destroyed stapes footplate), primary reconstruction of the ossicular chain was not performed in nine patients.

\section{a. HRQOL measurement}

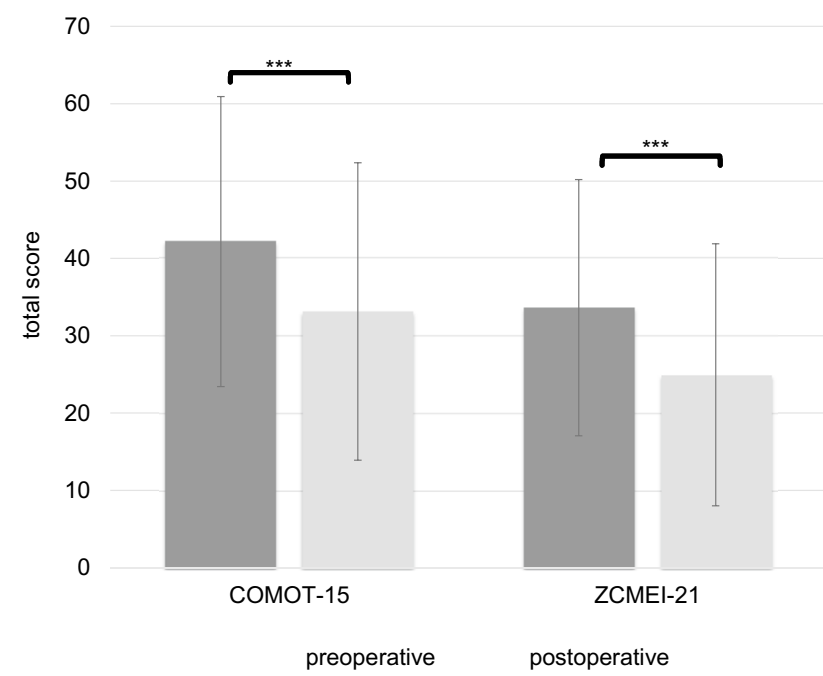

Fig. 1 COMOT-15 and ZCMEI-21 total score: comparison of preand postoperative results $(n=102)$; Data shown are mean \pm SD; $* * * p \leq 0.001$ preoperative vs. postoperative

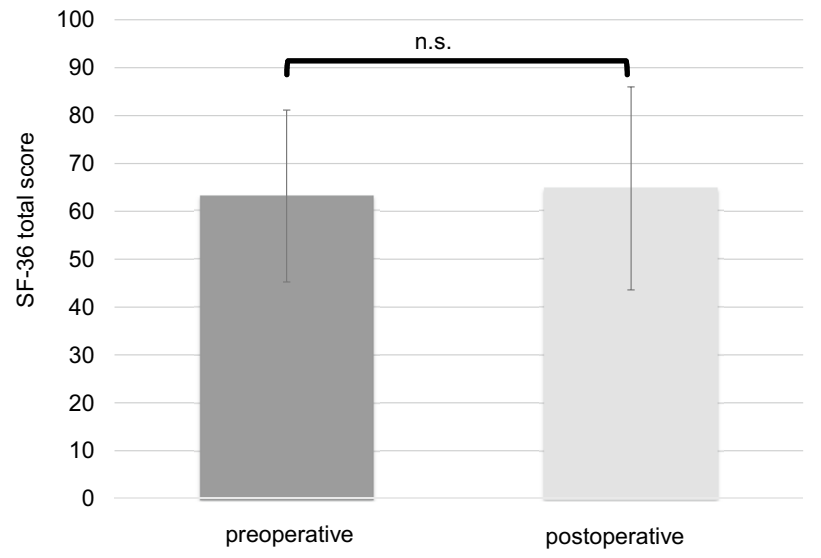

Fig. 2 SF-36 total score: comparison of pre- and postoperative results $(n=102)$; Data shown are mean \pm SD; $n$.s. not significant $(p>0.05)$

Middle ear surgery resulted in significantly better total COMOT-15 and ZCMEI-21 scores postoperatively (Fig. 1). However, when comparing pre- and postoperative results, no significant change in the total SF-36 score was found (Fig. 2).

b. Impact of postoperative hearing level on postoperative $H R Q O L$

The mean preoperative AC was $48.9 \pm 22.5 \mathrm{~dB}$, and this changed to $47.1 \pm 23.8 \mathrm{~dB}$ postoperatively $(p>0.05)$. Eleven patients $(10.8 \%)$ had a postoperative $\mathrm{AC}$ of $20 \mathrm{~dB}$ or lower, $34.3 \%$ (35) patients an $\mathrm{AC}$ of 21-40 dB, 26.5\% (27) an AC of 41-60 dB, and 28.4\% (29) an $A C$ of $>60 \mathrm{~dB}$. No significant difference was found between the pre- and postoperative bone conduction thresholds (mean difference $-2.9 \pm 8.8 \mathrm{~dB}$, $p>0.05$ ).

Correlation analyses were performed between both the postoperative COMOT-15 and ZCMEI-21 scores and postoperative AC. A moderate association between the postoperative audiometric data and the postoperative overall scores of COMOT-15 and ZCMEI-21 was observed (Fig. 3).

c. Impact of depressiveness on HRQOL

Generally, depressiveness measured by PHQ-9 scores did not change after middle ear surgery (mean difference $0.9, p>0.05)$. Of the participants, $31(30.1 \%)$ with COM had a PHQ-9 score of 5 or greater, indicating elevated depressive symptoms preoperatively. The mean postoperative COMOT-15 and ZCMEI-21 scores were significantly higher in patients with preoperative depressive symptoms than in patients without depressive symptoms $(p<0.001$, Fig. 4). Patients with preoperative depressive symptoms reported significantly lower SF-36 scores, indicating worse general HRQOL in the postoperative setting (Fig. 4).

d. Multivariate analysis 

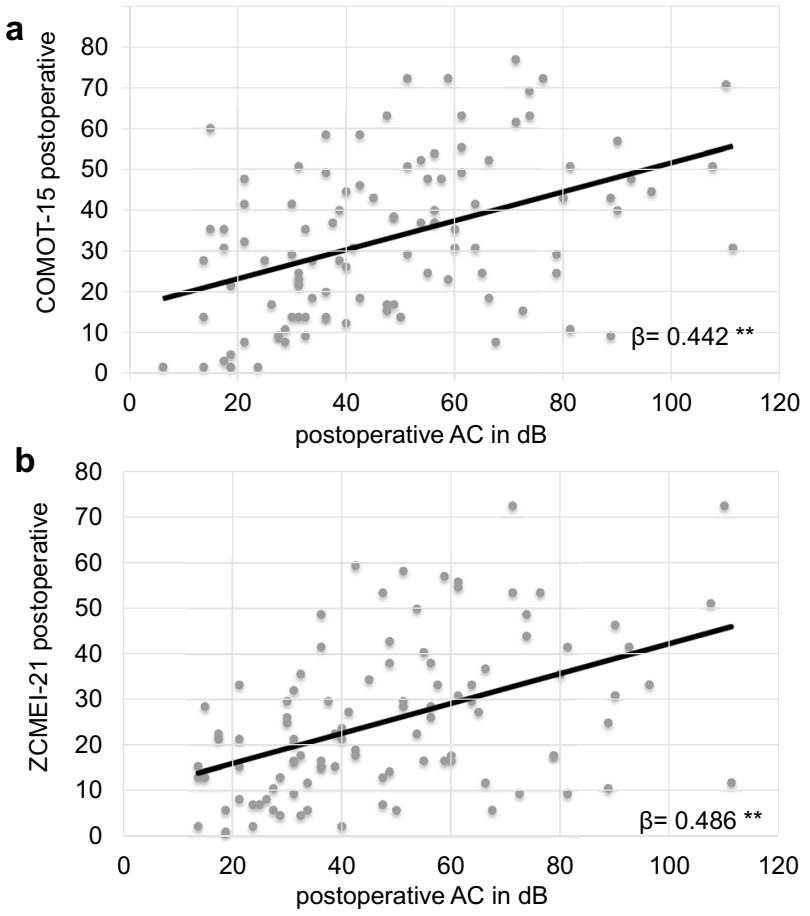

Fig. 3 Postoperative assessment of COMOT-15 (a) and ZCMEI-21 (b): bivariate regression between total scores and postoperative air conduction threshold (AC); $\beta$ regression coefficient, ${ }^{* *} p \leq 0.01$ )

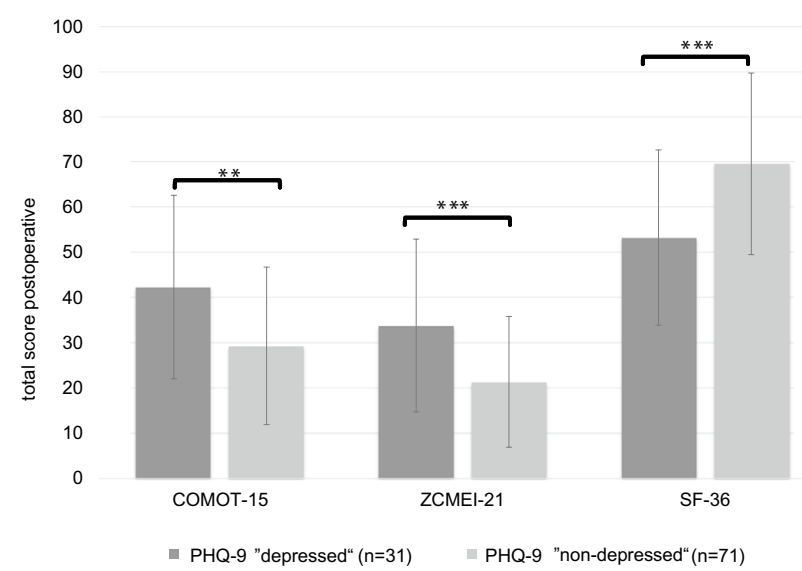

Fig. 4 Postoperative assessment of COMOT-15, ZCMEI-21 and SF-36: comparison of preoperative depressed versus non-depressed patients on the categorical PHQ-9; PHQ-9 nine-item depression scale of the patient health questionnaire; $*^{* *} p \leq 0.001$ depressed versus non-depressed; $* * * p \leq 0.01$ depressed versus non-depressed

Multivariate analyses for postoperative disease-specific HRQOL were performed with explanatory variables including middle ear status, comorbidity, depressiveness, and hearing improvement. The results of the multiple regression analyses showed that, even when adjusting for the OOPS index, CCI, and improvement in $\mathrm{AC}$, preoperative depressiveness remained significantly associated with higher COMOT-15 (Fig. 5) and ZCMEI-21 (Fig. 6) scores postoperatively. If the hearing improvement (change in $\mathrm{AC}$ ) was replaced by the postoperative $\mathrm{AC}$ in this regression model, the effect was almost the same (data not shown).

\section{Discussion}

Satisfactory surgical, functional, and HRQOL outcomes have been well documented after reconstructive middle ear surgery [3, 4, 8-11, 32, 33]. However, so far almost all studies have neglected the psychopathological predisposition of patients.

In Germany, the prevalence of current depressive symptoms is approximately $8.1 \%$ (women: $10.2 \%$; men: $6.1 \%$ ) [34]. In our survey of 102 patients with COM, 31 patients presented with clinically relevant depressive symptoms. Depression rates (indicated by PHQ responses) in COM patients are similar to those of chronic disease populations. Similar results were obtained in rhinological studies elucidating the prevalence of depressive disorders in patients with chronic rhinosinusitis [35].

Depression is associated with various chronic medical illnesses, including coronary heart disease and diabetes [36]. The bidirectional relationship between a chronic disease and comorbid depression hinders unequivocal assessment of causality. However, it has been proven that comorbid depression negatively influences treatment outcomes. Patients with depressive disorders were shown to have a higher risk of adverse outcomes, increased analgesic consumption, and increased use of the healthcare system [20]. In analyzing the post-interventional outcome in patients with chronic diseases, such as degenerative lumbar spine disease, previous studies indicated a strong interaction between preoperative impaired mental health status and patient-reported outcome $[17,18]$. In otorhinolaryngology head and neck surgery (ORL HNS), the association between psychological comorbidities and post-treatment outcome has been minimally investigated. The coexistence of depressive disorders and head and neck cancer [37, 38], chronic rhinosinusitis [39, 40], allergic disorders [41], tinnitus [42], and hearing disorders [12-14] is well described. However, the impact of psychological burden on PROMs after treatment was assessed in only a few studies. In patients with chronic rhinosinusitis, the preoperative disease burden was higher in patients with depressive disorders. Additionally, postoperative improvement in disease-specific HRQOL after endoscopic sinus surgery was significantly lower in patients with depressive 
Fig. 5 Multiple regression analysis of influencing factors on postoperative COMOT-15; CCI Charlson comorbidity index; OOPS ossiculoplasty outcome staging index; AC, air conduction threshold; Data shown are mean $\pm \mathrm{SD} ; \beta$ regression coefficient; $* * * p \leq 0.001$

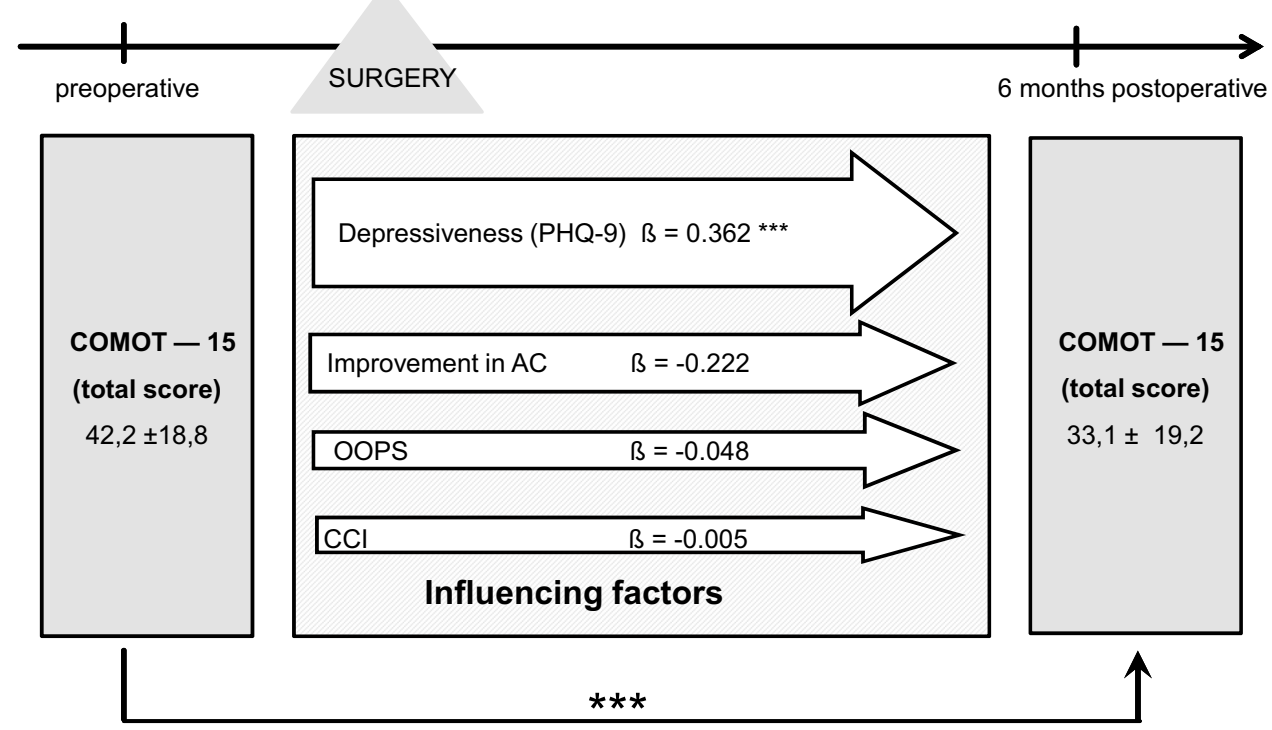

Fig. 6 Multiple regression analysis of influencing factors on postoperative ZCMEI-21; $C C I$, Charlson comorbidity index; OOPS ossiculoplasty outcome staging index; $A C$ air conduction threshold; Data shown are mean $\pm \mathrm{SD} ; \beta$ regression coefficient; *** $p \leq 0.001$; ${ }^{*} p \leq 0.05$

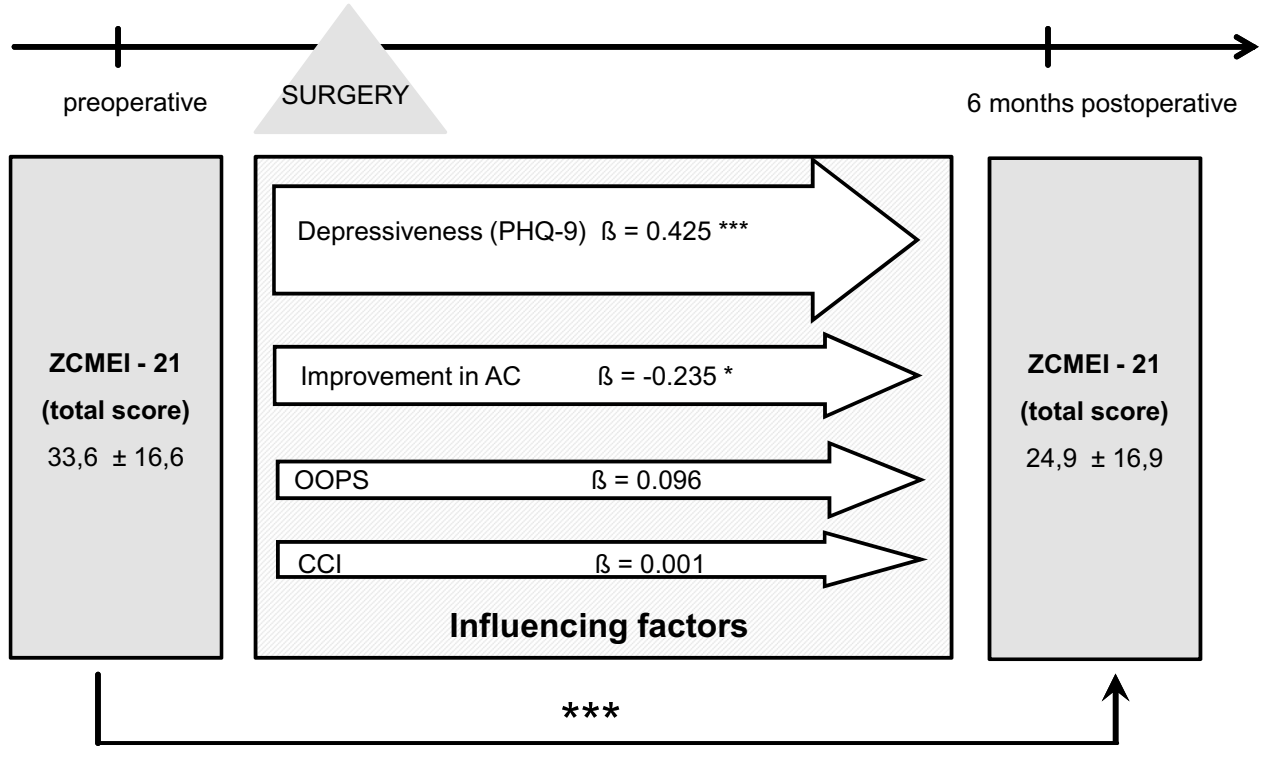

symptoms [43]. In patients undergoing cochlear implantation, HRQOL was shown to be influenced by depressive symptoms, anxiety, and the perception of stress [44, 45].

In this study, we observed a general significant improvement in disease-specific HRQOL after middle ear surgery. Postoperative HRQOL correlated moderately with the postoperative AC, which is consistent with former HRQOL analyses [3, 8, 11]. However, the results of our multiple regression models stress the importance of preoperative depressiveness as the main predictor of postoperative HRQOL after middle ear surgery. Furthermore, otological studies mainly addressed pathology and surgery-related factors that may influence the HRQOL outcome. Univariate analyses of potential influencing factors, such as the presence of cholesteatoma, surgical techniques, revision surgery, mastoid obliteration, and bilateral disease, showed heterogeneous results [3, 10, 11, 46, 47]. Considering these results, patients with normal postoperative clinical observations (intact tympanic membrane, dry ear, and sufficient improvement in AC) and a low disease-specific HRQOL should be screened for concomitant depressive symptoms and, if necessary, offered a specific supportive therapy.

Previously, minimal attention has been devoted to managing depressive disorders in patients with COM and investigating how the management of depressive disorders may affect COM-specific HRQOL after surgery. However, our results suggest that modulation of mood disorders may open new doors to improve HRQOL in COM patients. It 
may be important to address comorbid depressive disorders as a component of comprehensive management of patients with increased depressiveness and COM. In the presence of manifest psychological disorder, appropriate therapeutic cooperation should, therefore, be discussed even before surgery. Our results support the need for interventional studies to test and confirm this hypothesis.

In ORL HNS, the feasibility and benefit of accompanying psychotherapy was mainly analyzed in cancer treatments [48]. Psychological intervention enables psychological counseling and support, increases psychological perseverance, and reduces negative psychological and adverse social reactions $[49,50]$. To analyze the effect of psychological intervention on postoperative outcomes, high-quality, prospective trials are required with baseline psychological evaluation, standardized interventions, and integrated reporting of quality of life, psychological, and physiological outcome measures [46]. Reconstructive orthopedic studies, which analyzed the effectiveness of psychological intervention in hip or knee arthroplasty, showed heterogeneous results in terms of functional outcome and pain control [51]. However, the quality of prior studies has not been ideal due to the lack of long-term outcome and the ignored demand effect. Presumably, patients with increased depressiveness may benefit more from psychological intervention than those without an impaired mental health status [51]. So far, no similar investigations have been published for non-oncological ORL HNS.

General HRQOL remained stable over the observation period and did not improve significantly after middle ear surgery in our study. These results are consistent with those of other studies, which analyzed general HRQOL after ear surgery [3, 4]. Specific symptoms that may affect everyday life are insufficiently represented by general HRQOL measurement instruments. However, general HRQOL instruments are necessary to measure the impact of specific diseases on general health and allow comparisons that determine the impact of different diseases on general HRQOL [3].

While the data were prospectively collected, only a few patient demographics and comorbidities were included in our data. We may have failed to capture other factors that are important in patients' perception of COM and its treatment. Our psychosocial assessment mainly focused on depressive symptoms. No other psychiatric or psychosomatic disturbances were analyzed. Particularly, the impact of anxiety, personality, and somatization should be investigated in more detail in further investigations, as studies from other subject areas demonstrated possible effects on PROMs [20, 23]. Furthermore, we must acknowledge a selection bias, as only symptomatic patients underwent middle ear surgery and were included. Asymptomatic patients are not usually sent to our tertiary referral center. Therefore, it was impossible to provide an adequate control group. Additionally, it is noteworthy that the study did not consider previous diagnoses of a depressive disorder or past medical treatment or psychotherapy. The extent of depressiveness was determined by a self-report inventory and was not supplemented by a psychological examination of a psychiatrist or clinical psychologist. Diagnosis of depression has traditionally been based on clinical criteria, including patients' symptoms and history. However, to standardize data and interpretations, various interview-based instruments for depression screening were developed and used in both clinical and research practice. For the diagnosis of depressive disorder, the PHQ-9 showed excellent criterion validity in medical patients, with a sensitivity of $95 \%$ and a specificity of $86 \%$ [28]. It takes approximately $5 \mathrm{~min}$ to complete the questionnaire. Evaluation of the PHQ-9 takes approximately 2 min. Diagnostic validity and easy application make it a practicable tool even in busy clinical practice settings.

In the perioperative setting, the evaluation of patientrelated factors and how they influence the patient's surgical candidacy is ever more crucial in value-based care models. Depressiveness is a pertinent comorbidity that should be included in that evaluation. Consequently, we recommend routine preoperative screening of COM patients for depressiveness to initiate differentiated psychological supportive care.

\section{Conclusion}

Depressive disorders occur with increased prevalence in patients with COM. The presence of comorbid depressiveness with COM leads to poorer HRQOL outcomes after reconstructive middle ear surgery. Therefore, improvements are required in screening, diagnosis, and treatment for patients with comorbid depressive disorders and COM. Simultaneously, if no improvement is observed in the measured HRQOL, an unknown depressive symptomatology must be considered if a clear clinical improvement is observed in the disease symptoms.

Funding Open Access funding enabled and organized by Projekt DEAL.

Code availability Not applicable.

\section{Compliance with ethical standards}

Conflict of interest All authors reported no conflicts of interest.

Ethical standards All procedures performed in studies involving human participants were in accordance with the ethical standards of local ethi- 
cal review committee (EK 166042017) and with the 1964 Helsinki declaration and its later amendments or comparable ethical standards.

Informed consent Informed consent was obtained from all the participants.

Availability of data and material The datasets generated during and/ or analysed during the current study are not publicly available, but are available from the corresponding author on reasonable request.

Open Access This article is licensed under a Creative Commons Attribution 4.0 International License, which permits use, sharing, adaptation, distribution and reproduction in any medium or format, as long as you give appropriate credit to the original author(s) and the source, provide a link to the Creative Commons licence, and indicate if changes were made. The images or other third party material in this article are included in the article's Creative Commons licence, unless indicated otherwise in a credit line to the material. If material is not included in the article's Creative Commons licence and your intended use is not permitted by statutory regulation or exceeds the permitted use, you will need to obtain permission directly from the copyright holder. To view a copy of this licence, visit http://creativecommons.org/licenses/by/4.0/.

\section{References}

1. Kaftan H, Noack M, Friedrich N et al (2008) Prevalence of chronic tympanic membrane perforation in the adult population. HNO 56:145-150. https://doi.org/10.1007/s00106-007-1574-0

2. Browning GG, Gatehouse S (1992) The prevalence of middle ear disease in the adult British population. Clin Otolaryngol Allied Sci 17:317-321. https://doi.org/10.1111/j.1365-2273.1992. tb01004.x

3. Baumann I, Gerendas B, Plinkert PK, Praetorius M (2011) General and disease-specific quality of life in patients with chronic suppurative otitis media-a prospective study. Health Qual Life Outcomes 9:48. https://doi.org/10.1186/1477-7525-9-48

4. Nadol JB, Staecker H, Gliklich RE (2000) Outcomes assessment for chronic otitis media: the chronic ear survey. The Laryngoscope 110:32-35. https://doi.org/10.1097/00005537-20000 3002-00009

5. Phillips JS, Haggard M, Yung M (2014) A new health-related quality of life measure for active chronic otitis media (COMQ12): development and initial validation. Otol Neurotol 35:454458. https://doi.org/10.1097/MAO.0000000000000205

6. Homøe P, Heidemann CH, Damoiseaux RA et al (2020) Panel 5: Impact of otitis media on quality of life and development. Int J Pediatr Otorhinolaryngol 130(Suppl 1):109837. https://doi. org/10.1016/j.ijporl.2019.109837

7. Neudert M (2020) Quality in middle ear surgery - a critical position determination. Laryngorhinootologie 99:S222-S271. https://doi.org/10.1055/a-1021-6427

8. Baumann I, Kurpiers B, Plinkert PK, Praetorius M (2009) Development and validation of the chronic otitis media outcome test 15 (COMOT-15). Measurement of health-related quality of life in patients with chronic otitis media. HNO 57:889-895. https://doi.org/10.1007/s00106-008-1870-3

9. Bächinger D, Röösli C, Ditzen B, Huber AM (2016) Development and validation of the Zurich chronic middle ear inventory (ZCMEI-21): an electronic questionnaire for assessing quality of life in patients with chronic otitis media. Eur Arch OtoRhino-Laryngol 273:3073-3081. https://doi.org/10.1007/s0040 5-016-3915-7
10. Choi SY, Cho Y-S, Lee NJ et al (2012) Factors associated with quality of life after ear surgery in patients with chronic otitis media. Arch Otolaryngol Head Neck Surg 138:840-845. https ://doi.org/10.1001/archoto.2012.1800

11. Lailach S, Kemper M, Lasurashvili N et al (2015) Health-related quality of life measurement after cholesteatoma surgery: comparison of three different surgical techniques. Eur Arch OtoRhino-Laryngol 272:3177-3185. https://doi.org/10.1007/s0040 5-014-3370-2

12. Cosh S, Carriere I, Delcourt C et al (2020) A dimensional approach to understanding the relationship between selfreported hearing loss and depression over 12 years: the threecity study. Aging Ment Health. https://doi.org/10.1080/13607 863.2020.1727845

13. Golub JS, Brewster KK, Brickman AM et al (2020) Subclinical hearing loss is associated with depressive symptoms. Am J Geriatr Psychiatry. https://doi.org/10.1016/j.jagp.2019.12.008

14. Dawes P, Emsley R, Cruickshanks KJ et al (2015) Hearing loss and cognition: the role of hearing AIDS, social isolation and depression. PLoS ONE 10:e0119616. https://doi.org/10.1371/ journal.pone.0119616

15. Bakir S, Kinis V, Bez Y et al (2013) Mental health and quality of life in patients with chronic otitis media. Eur Arch Oto-Rhino-Laryngol 270:521-526. https://doi.org/10.1007/s00405-012-2031-6

16. Mehboob S, Rafi ST, Ahmed N, Mehjabeen N (2019) Association of hearing loss with depression, anxiety and stress in patients suffering from Chronic Suppurative Otitis Media. Pak J Med Sci 35:510-514. https://doi.org/10.12669/pjms.35.2.152

17. Lebow R, Parker SL, Adogwa O et al (2012) Microdiscectomy improves pain-associated depression, somatic anxiety, and mental well-being in patients with herniated lumbar disc. Neurosurgery 70:306-311

18. Chaichana KL, Mukherjee D, Adogwa O et al (2011) Correlation of preoperative depression and somatic perception scales with postoperative disability and quality of life after lumbar discectomy. J Neurosurg Spine 14:261-267. https://doi. org/10.3171/2010.10.SPINE10190

19. Heary KO, Wong AWK, Lau SCL et al (2020) Quality of Life and psychosocial factors as predictors of pain relief following nerve surgery. Hand N Y N. https://doi.org/10.1177/15589 44720911213

20. Harris AB, Marrache M, Puvanesarajah V et al (2020) Are preoperative depression and anxiety associated with patientreported outcomes, health care payments, and opioid use after anterior discectomy and fusion? Spine. https://doi. org/10.1016/j.spinee.2020.03.004

21. Schultz BJ, Tanner N, Shapiro LM et al (2020) Patientreported outcome measures (PROMs): influence of motor tasks and psychosocial factors on faam scores in foot and ankle trauma patients. J Foot Ankle Surg. https://doi.org/10.1053/j. jfas.2020.01.008

22. Rahman R, Ibaseta A, Reidler JS et al (2020) Changes in patients' depression and anxiety associated with changes in patient-reported outcomes after spine surgery. J Neurosurg Spine. https://doi.org/10.3171/2019.11.SPINE19586

23. Nowicka-Sauer K, Hajduk A, Kujawska-Danecka H et al (2018) Illness perception is significantly determined by depression and anxiety in systemic lupus erythematosus. Lupus 27:454-460. https://doi.org/10.1177/0961203317751858

24. Rogante E, Sarubbi S, Lamis DA et al (2019) Illness perception and job satisfaction in patients suffering from migraine headaches: trait anxiety and depressive symptoms as potential mediators. Headache 59:46-55. https://doi.org/10.1111/head.13461

25. Sirri L, Pierangeli G, Cevoli S et al (2018) Illness perception in patients with migraine: an exploratory study in a tertiary 
care headache centre. J Psychosom Res 111:52-57. https://doi. org/10.1016/j.jpsychores.2018.05.011

26. Bullinger $M$ (1995) German translation and psychometric testing of the SF-36 Health Survey: Preliminary results from the IQOLA project. Soc Sci Med 41:1359-1366. https://doi. org/10.1016/0277-9536(95)00115-N

27. Ware JE, Kosinski M, Keller SD et al (1994) SF-36 physical and mental health summary scales: a user's manual. The Health Institute, New England Medical Center, MA

28. Gräfe K, Zipfel S, Herzog W (2010) Löwe screening psychischer störungen mit dem gesundheitsfragebogen für patienten (PHQ-D). Diagnostica 1:171-181

29. Quan H, Li B, Couris CM et al (2011) Updating and validating the Charlson comorbidity index and score for risk adjustment in hospital discharge abstracts using data from 6 countries. Am J Epidemiol 173:676-682. https://doi.org/10.1093/aje/kwq433

30. Dornhoffer JL, Gardner E (2001) Prognostic factors in ossiculoplasty: a statistical staging system. Otol Neurotol 22:299-304

31. Committee on Hearing and Equilibrium (1995) Committee on hearing and equilibrium guidelines for the evaluation of results of treatment of conductive hearing loss. Otolaryngol-Head Neck Surg 113:186-187

32. Dornhoffer JL, Smith J, Richter G, Boeckmann J (2008) Impact on quality of life after mastoid obliteration. The Laryngoscope 118:1427-1432. https://doi.org/10.1097/MLG.0b013e3181 73dd $7 \mathrm{e}$

33. Lucidi D, De Corso E, Paludetti G, Sergi B (2019) Quality of life and functional results in canal wall down vs canal wall up mastoidectomy. Acta Otorhinolaryngol Ital 39:53-60

34. Busch MA, Maske UE, Ryl L et al (2013) Prevalence of depressive symptoms and diagnosed depression among adults in Germany: results of the German Health Interview and examination survey for Adults (DEGS1). Bundesgesundheitsblatt Gesundheitsforschung Gesundheitsschutz 56:733-739. https://doi. org/10.1007/s00103-013-1688-3

35. Vandelaar LJ, Jiang ZY, Saini A et al (2020) PHQ-9 and SNOT22: elucidating the prevalence of depression in chronic rhinosinusitis. Otolaryngol-Head Neck Surg 162:142-147. https://doi. org/10.1177/0194599819886852

36. van der Zwaan GL, van Dijk SEM, Adriaanse MC et al (2016) Diagnostic accuracy of the patient health questionnaire-9 for assessment of depression in type II diabetes mellitus and/or coronary heart disease in primary care. J Affect Disord 190:68-74. https://doi.org/10.1016/j.jad.2015.09.045

37. Gosak M, Gradišar K, Rotovnik Kozjek N, Strojan P (2020) Psychological distress and nutritional status in head and neck cancer patients: a pilot study. Eur Arch Oto-Rhino-Laryngol 277:12111217. https://doi.org/10.1007/s00405-020-05798-y

38. Henry M, Fuehrmann F, Hier M et al (2019) Contextual and historical factors for increased levels of anxiety and depression in patients with head and neck cancer: a prospective longitudinal study. Head Neck 41:2538-2548. https://doi.org/10.1002/ hed. 25725

39. Mattos JL, Ashby S, Payne SC et al (2020) Pilot investigation: prospective needs assessment of knowledge, attitude, and insight about mental health treatment options in patients with chronic rhinosinusitis. Am J Rhinol Allergy. https://doi.org/10.1177/19458 92420912369

40. Speth MM, Phillips KM, Hoehle LP et al (2019) Longitudinal improvement in nasal obstruction symptoms of chronic rhinosinusitis directly associates with improvement in mood. Eur Arch Oto-Rhino-Laryngol 276:2827-2833. https://doi.org/10.1007/ s00405-019-05569-4

41. Lu Z, Chen L, Xu S et al (2018) Allergic disorders and risk of depression: a systematic review and meta-analysis of 51 largescale studies. Ann Allergy Asthma Immunol 120:310-317.e2. https://doi.org/10.1016/j.anai.2017.12.011

42. Bhatt JM, Bhattacharyya N, Lin HW (2017) Relationships between tinnitus and the prevalence of anxiety and depression. The Laryngoscope 127:466-469. https://doi.org/10.1002/ lary. 26107

43. Ospina J, Liu G, Crump T et al (2019) The impact of comorbid depression in chronic rhinosinusitis on post-operative sino-nasal quality of life and pain following endoscopic sinus surgery. J Otolaryngol Head Neck Surg 48:18. https://doi.org/10.1186/s4046 3-019-0340-0

44. Davis A, McMahon CM, Pichora-Fuller KM et al (2016) Aging and hearing health: the life-course approach. The Gerontologist 56(Suppl 2):S256-267. https://doi.org/10.1093/geront/gnw033

45. Knopke S, Häussler S, Gräbel S et al (2019) Age-dependent psychological factors influencing the outcome of cochlear implantation in elderly patients. Otol Neurotol 40:e441-e453. https://doi. org/10.1097/MAO.0000000000002179

46. Levent Demir U, Yuksel Akyildiz M, Alpay M (2012) The factors which affect disease-specific quality of life in patients with chronic otitis media. J Int Adv Otol 8:371-378

47. Weiss NM, Bächinger D, Botzen J et al (2020) Mastoid cavity obliteration leads to a clinically significant improvement in healthrelated quality of life. Eur Arch Oto-Rhino-Laryngol. https://doi. org/10.1007/s00405-020-05881-4

48. Richardson AE, Broadbent E, Morton RP (2019) A systematic review of psychological interventions for patients with head and neck cancer. Support Care Cancer 27:2007-2021. https://doi. org/10.1007/s00520-019-04768-3

49. Levett DZH, Grimmett C (2019) Psychological factors, prehabilitation and surgical outcomes: evidence and future directions. Anaesthesia 74(Suppl 1):36-42. https://doi.org/10.1111/ anae. 14507

50. Peerbhoy D, Hall GM, Parker C et al (1982) (1998) Patients' reactions to attempts to increase passive or active coping with surgery. Soc Sci Med 47:595-601. https://doi.org/10.1016/s0277 -9536(98)00118-x

51. Bay S, Kuster L, McLean N et al (2018) A systematic review of psychological interventions in total hip and knee arthroplasty. BMC Musculoskelet Disord 19:201. https://doi.org/10.1186/ s12891-018-2121-8

Publisher's Note Springer Nature remains neutral with regard to jurisdictional claims in published maps and institutional affiliations. 